\title{
Fixed Dental Prostheses on Reduced Periodontal Tissue Support: About a Case
}

\author{
Ait Mehdi. M*, Zenati. L, Mebarki. S, Kehila. Bourouis.Y \\ Mustapha Bacha Hospital, University 1, Benyoucef Benkhedda Algiers, Algeria \\ *Corresponding Author: Ait Mehdi. M, Mustapha Bacha Hospital, University 1, Benyoucef Benkhedda \\ Algiers, Algeria.
}

\begin{abstract}
Periodontitis is a multifactorial immuno-inflammatory pathology which results in the destruction and apical migration of the attachment system of the dental organ.

Dental mobility is symptoms of periodontal disease which can be aggravated by occlusal overload and have aesthetic, functional and psychological repercussions for the patient.

The aim of this work is to show through this clinical case the importance of the restraint in the treatment of teeth with reduced periodontium.

This clinical case illustrates the treatment of dental mobility with a compression bridge performed after periodontal healing and functional and aesthetic validation of the prosthetic project in order to: relieve the patient, ensure function and aesthetics and protect mobile teeth periodontal lesions by stabilizing them in favorable occlusal relationships.

It is a 60-year-old woman with total edentulousness of the mandible and partial edging of the maxilla (Class I modification 1 KENNEDY APPLEGATE) with mobility of the four incisors.

The therapeutic approach consists of combining scaling, occlusal adjustment and restraint by fixed prosthesis.

There are other alternatives to the extraction of weakened periodontal movable teeth provided that a wellconducted therapeutic protocol is necessarily followed in three stages: pre-prosthetic, prosthetic and postprosthetic treatments.
\end{abstract}

Keywords: Fixed dental prosthese; reduced periodontal tissue support; restraint.

\section{INTRODUCTION}

Periodontitis is an inflammation of deep periodontal tissue of; it can cause a loss of attachment which with the course of the disease can lead to dental mobility. [1]

When occlusal trauma is associated with periodontitis, it becomes an aggravating factor of destruction of periodontal tissues (cementum, demodontium, alveolar bones) which will lead to an enlargement of the demodontium with angular bone lesions and an increase in dental mobility. However, dental mobility due to occlusal trauma alone is only the result of an adaptation of the periodontium to occlusal overload. It is therefore possible to save the moving teeth by eliminating the causes of occlusal overload. [2]

Dental mobility can have aesthetic, functional and above all psychological repercussions for the patient.
The aim of this work is to show through this clinical case the importance of the restraint in the treatment of teeth with reduced periodontium.

\section{Clinical CaSe}

It is a 60 year old woman who came to our consultation for a prosthetic restoration for functional and aesthetic purposes and who was already wearing a total prosthesis on the mandible. The patient who seems cooperative and motivated has hypertension on the general health plan.

\section{- Clinical Examination}

During the interview, the patient was worried and embarrassed by the state of her teeth and hid them with her hand.

The clinical examination revealed an unsatisfactory oral hygiene with the presence of tartar on the dental surfaces. 
The maxillary intra-oral examination highlights: index $\mathrm{CAD}=26(\mathrm{C}=06 ; \mathrm{A}=20)$; MUHLEMAN degree II mobility in teeth: 1112 21 and 22; a rotation of 15; vestibuloversion of 112122 ; erosion of all teeth and the presence of inter-dental diastemas (Figure 1)

Periodontal evaluation revealed a large, generalized gingival recession in all of the remaining teeth. (Figure 2)

At the level of the mandible, there is a total absence of tooth with a degree of class II resorption according to ATWOOD. (Figure 3)

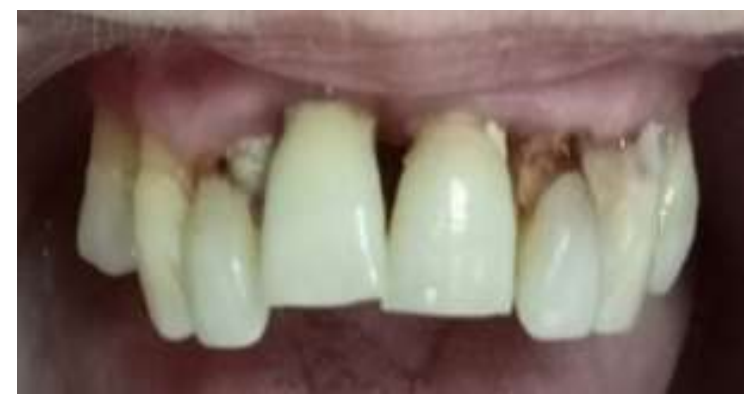

Figure1. Intraoral view

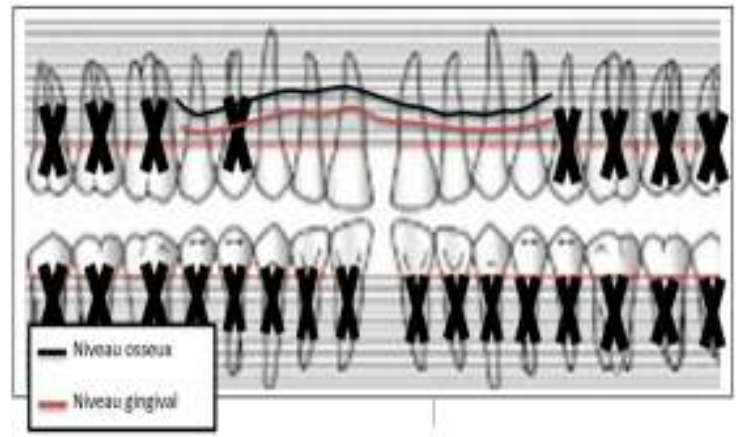

Figure2. Periodontal assessment

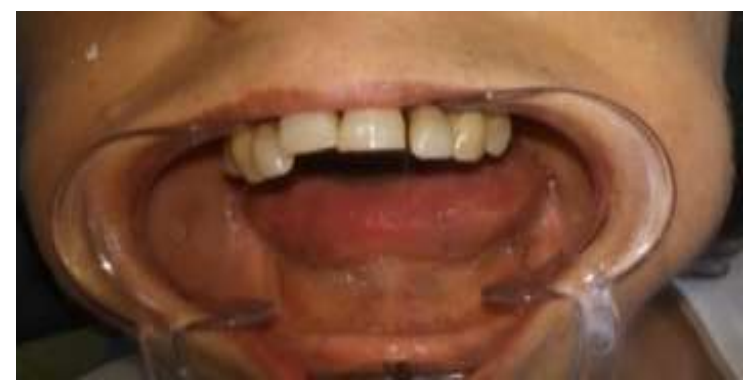

Figure3. Total toothlessness on the mandible (class II ATWOOD)

\section{- Radiological Examination}

The panoramic radiography supplemented by retro alveolar images allowed the analysis of the periodontal status which reveals an advanced generalized horizontal osseous lysis; with a desmodontal enlargement at the level of the central and lateral incisors. (Figures 4a; 4b; 4c)

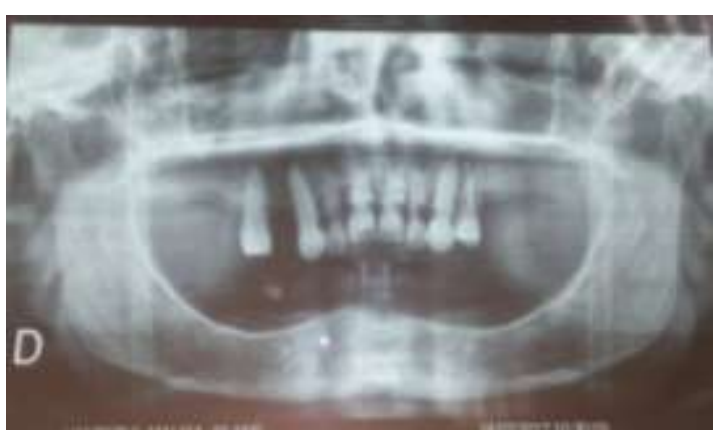

Figure 4a

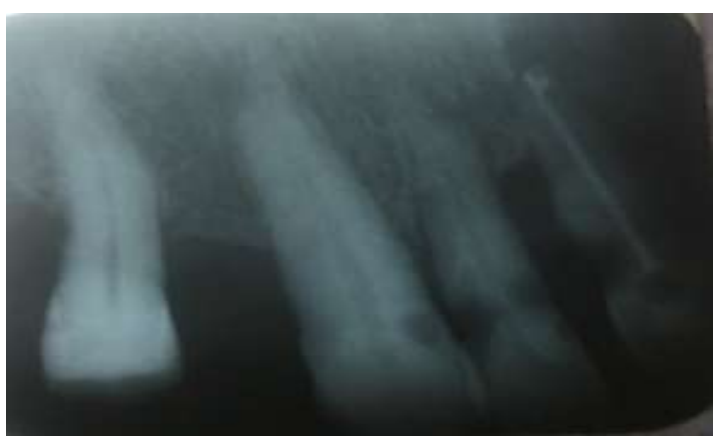

Figure 4b

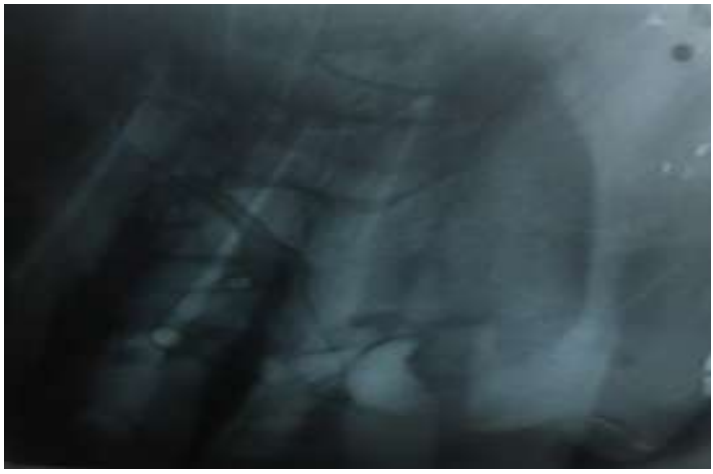

Figure 4c

Figure4a; 4b; 4c. Radiological examination showing horizontal bone lesions; caries on level $13 / 12 / 11 / 21 / 22 / 23$ and a widening of the periodontal level of the central and lateral incisor

\section{- Diagnostic}

It is an advanced generalized chronic periodontitis associated with a secondary occlusal trauma caused by the absence of posterior wedging (toothlessness not compensated)

\section{Processing of the Treatment}

\subsection{Preprosthetic Treatment}

The therapeutic approach consisted first of all in resolving periodontal problems by motivating oral hygiene and teaching plaque control methods (dental brushing). This motivation session was supplemented by scaling and surfacing. 
A prosthetic project with a diagnostic wax (wax up) was envisaged in order to preview the aesthetic result; to be able to manage possible interference and anticipate possible problems. (Figures: 5a; 5b; 5c; 5d; 5th; 5f)

Hygiene being satisfactory, endodontic care and strutted coronary-root reconstructions were undertaken on the four incisors due to the importance of the vestibulo-version.

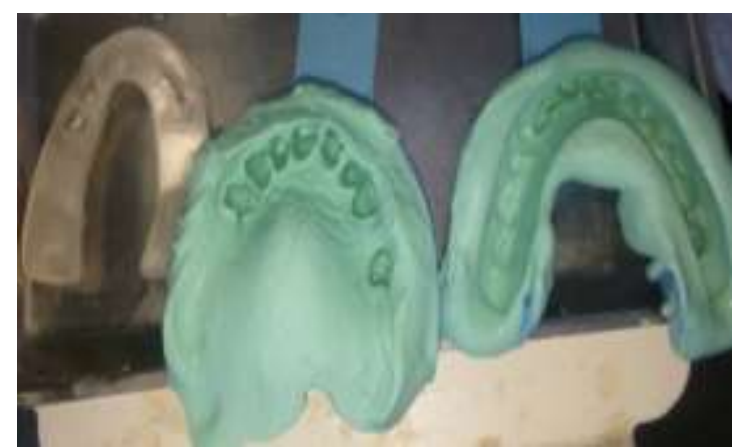

Figure5a. Preliminary alginate impressions

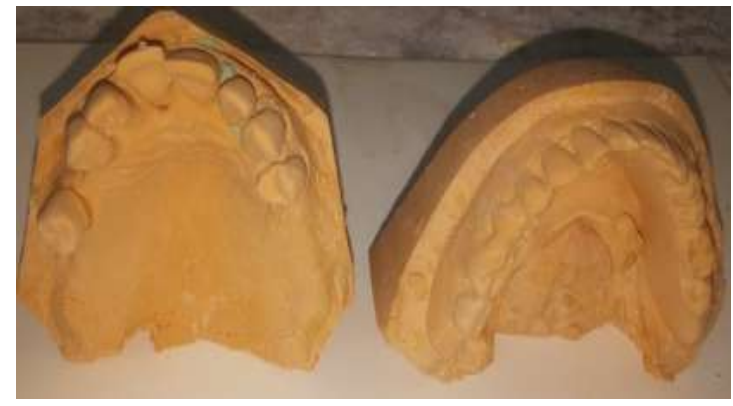

Figure5b. Casting of the study models

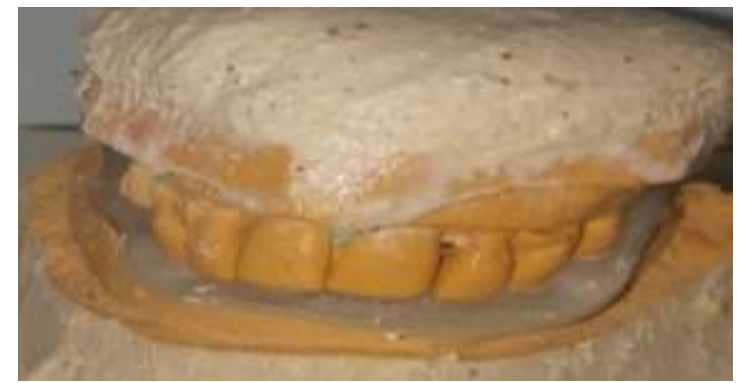

Figure5c. Placing in an occlude

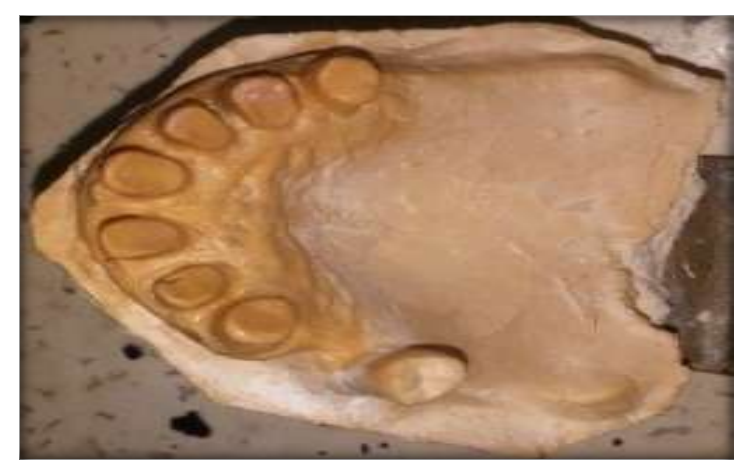

Figure 5d. peripheral corono preparations on study model (occlusal View)

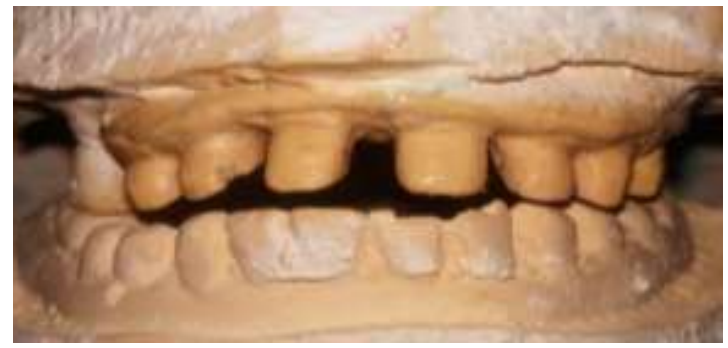

Figure5e. Peripheral corono preparations on study model (front view)

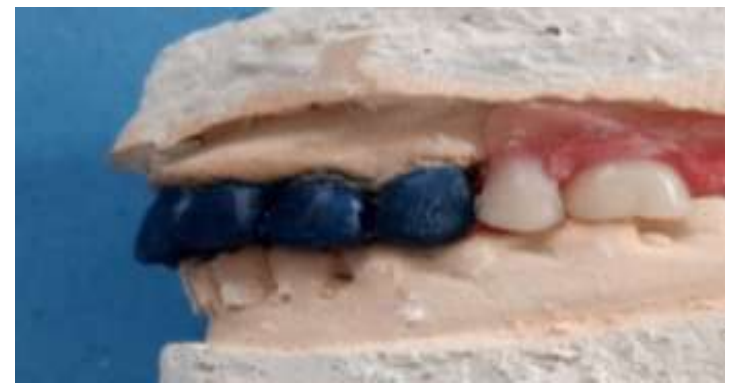

Figure5f. Validation of the prosthetic project

\subsection{Prosthetic Treatment}

After validation of the prosthetic project, the peripheral corono preparations could be carried out by recommending cervical limits slightly in intrasulular on the vestibular sides on the six anterior teeth in order to increase the esthetic yield and the retention of the bridge. Then we made a technical wash impression and finally temporary polycarbonate caps were adjusted, secured and provisionally sealed. (Figure 6a; 6b)

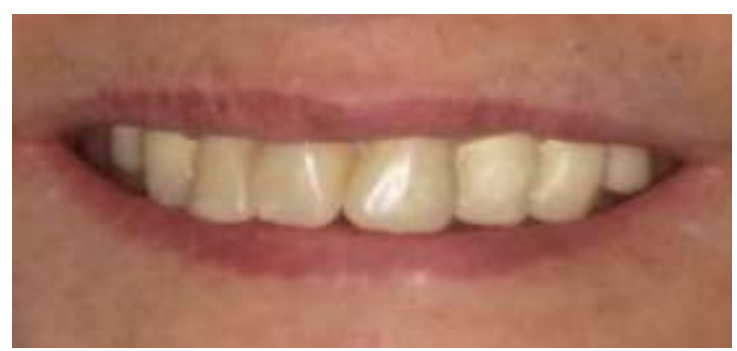

Figure6a. Temporary caps

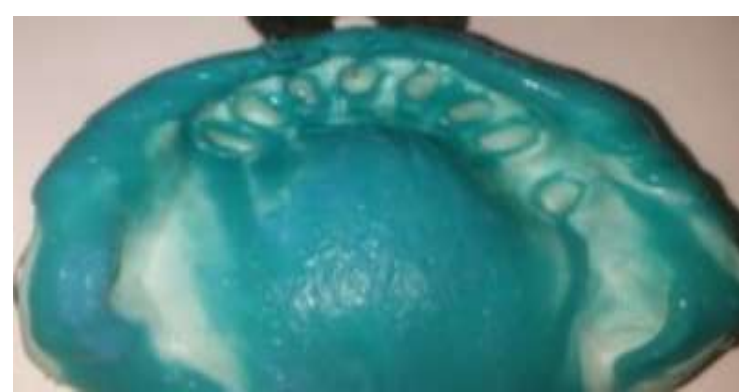

Figure6b. Impression taking (Wash Technic)

At the mandible, the classic stages of making a removable total prosthesis have been undertaken, namely: 
-The registration of the peripheral seal with Kerr's paste on an individual impression tray

-Realization of the secondary imprint to the $\mathrm{ZnO}$ eugenol paste (Figures: 7a; 7b)

- Recording of the piezographic prosthetic corridor

-Transfer of the superior working model on a semi-adaptable articulator (Quick Master) and recording of the occlusion in Centered Relation (Figures 8a; 8b; 8c; 8d).

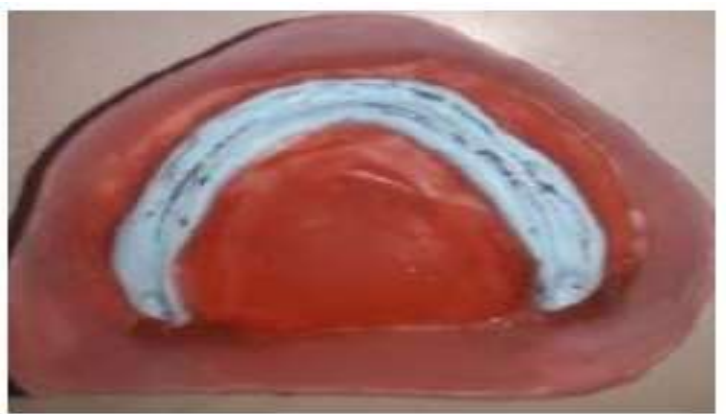

Figure7a. Peripheral seal and secondary impression to the $\mathrm{ZnO}$ eugenol paste of the mandibular arch

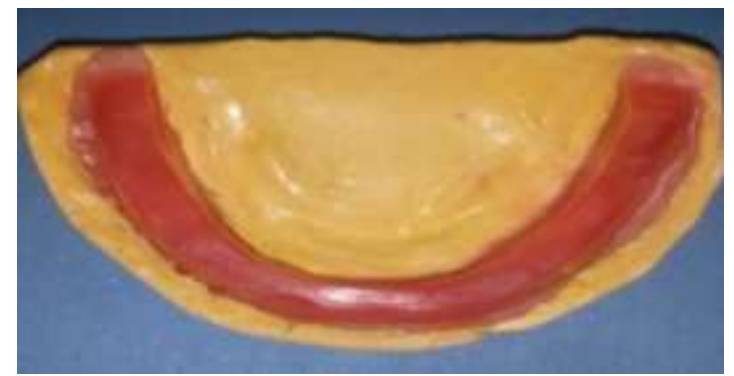

Figure7b. Piezographic duplicate occlusion wax

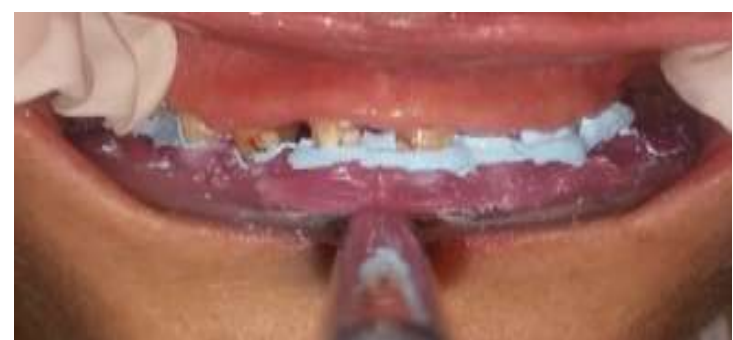

Figure8a. Recording of the position of the maxilla relative to the hinge axis

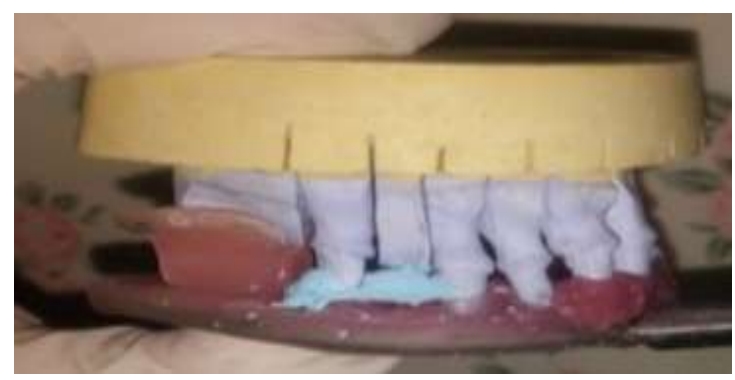

Figure8b. Installation of the upper model on a semi-adaptable articulator

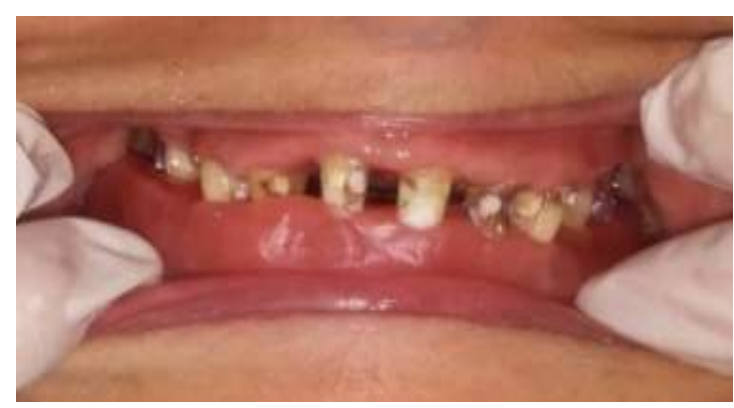

Figure8c. Registration of occlusion in $C R$

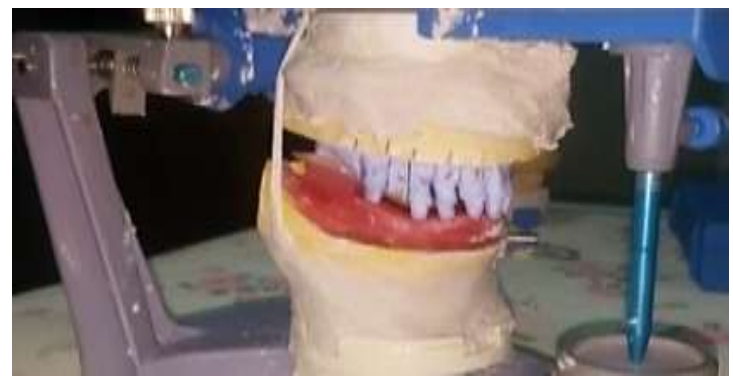

Figure8d. Fixing of the lower model on the lower branch of the semi-adaptable articulator

The framework of the future bridge was tested, taking care to check the precision of the adjustment of the edges of the framework on the limits of the preparations and to check the absence of compression at the level of the marginal gum by the contour of the framework. (absence of whitening of the marginal gum). (Figures 9a; 9b)

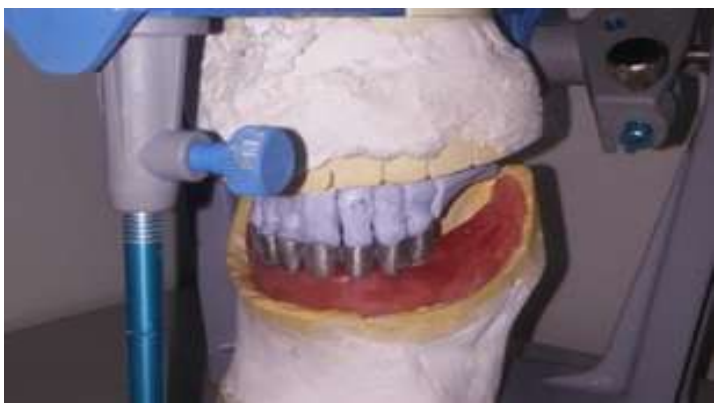

Figure9a. Bridge framework

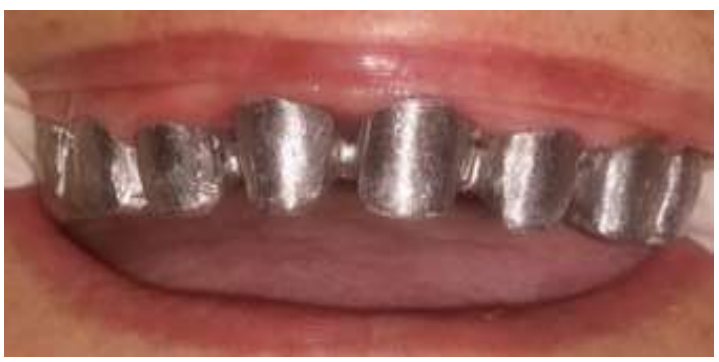

Figure9b. Test of the metallic framework absence of whitening of the marginal gum

Once the usage bridge was ready, the patient was reviewed for the functional test (Figures $10 \mathrm{a} ; 10 \mathrm{~b})$ 
The resin bridge was provisionally sealed (Figure 11) and finally the removable upper and lower acrylic resin prostheses were inserted after performing occlusal equilibration in bilaterally balanced occlusion (Figure 12)

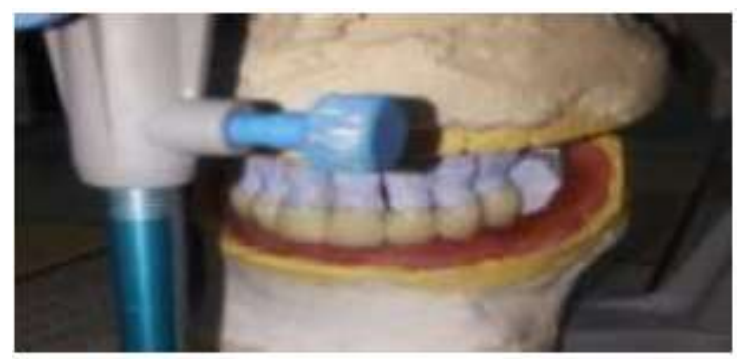

Figure10a. Bridge on articulator

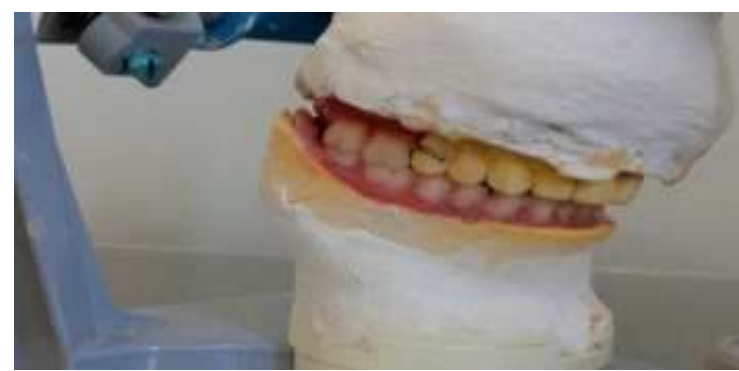

Figure10b. Mounting of artificial teeth on a semiadaptable articulator

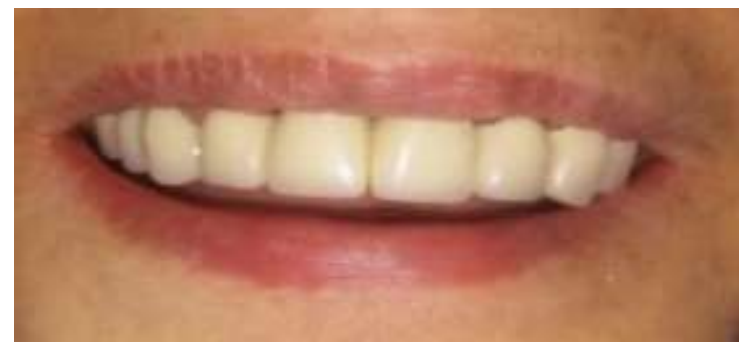

Figure11. Fitting of the use bridge and provisional sealing

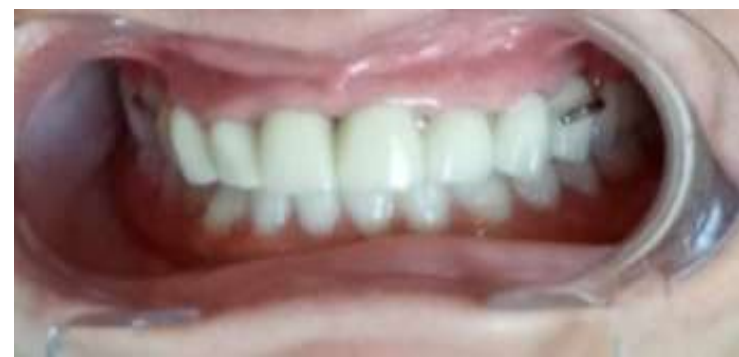

Figure12. Delivery of removable prosthese

\subsection{Post Prosthetic Treatment}

After the bridge was sealed, the patient was seen again at 6 months and 24 months for periodontal control and maintenance sessions, with alternating irrigations of all the gingivodental furrows with Chlorhexidine and hydrogen peroxide. We established an X-ray assessment at each inspection. (Figures 13a; 13b; 13c; 13d; 13e; 13f; 13g; 13h; 13i)

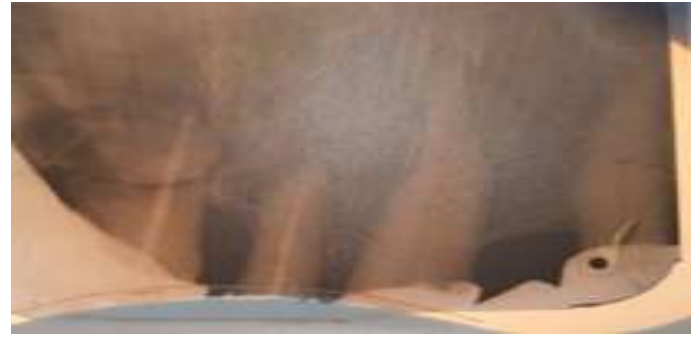

Figure 13a

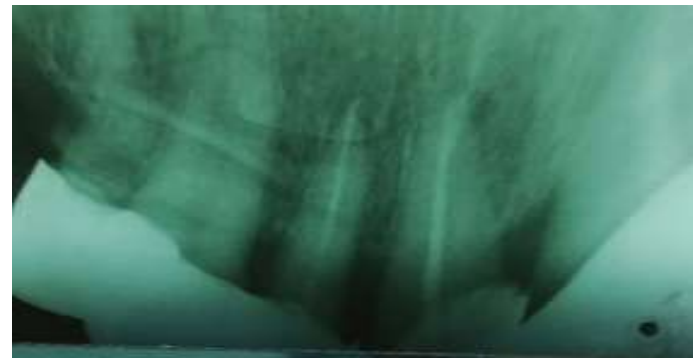

Figure 13b

Figure13a; 13b. Radiographic control 6 months after the placement of the compression bridge

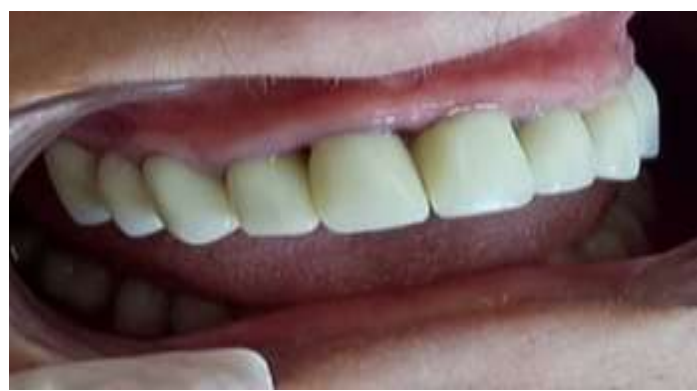

Figure 13c

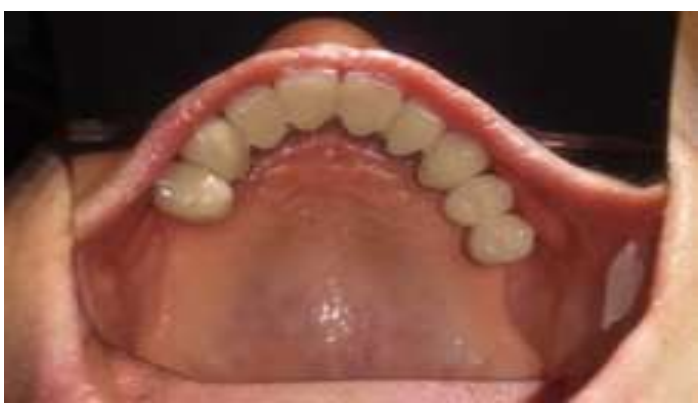

Figure 13d

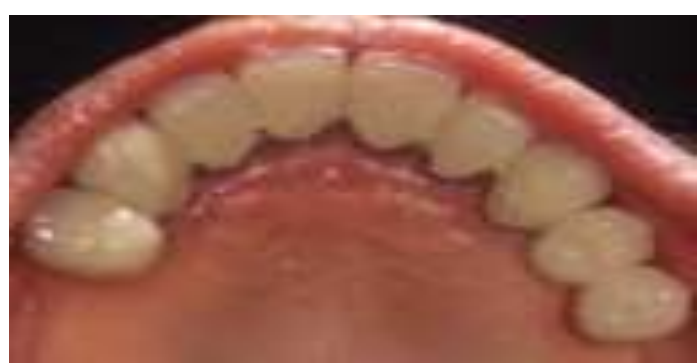

Figure 13e

Figure13c; 13d; 13e. Clinical control 06 months after the placement of the compression bridge 


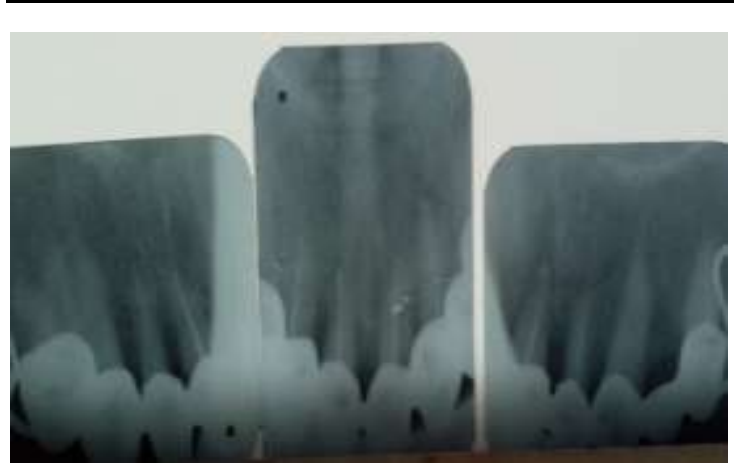

Figure13f. Radiological check 24 months after the placement of the compression bridge

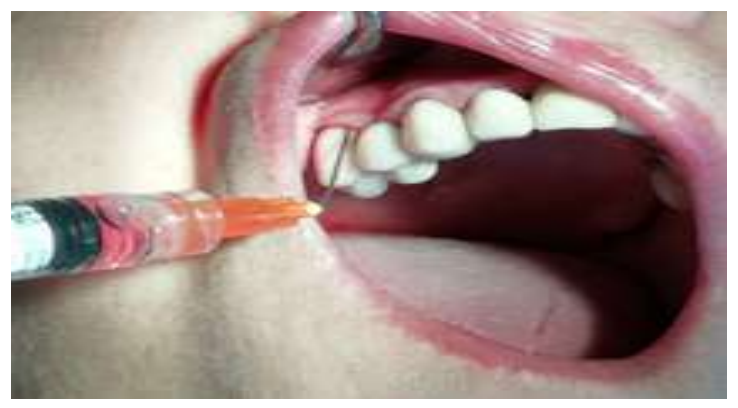

Figure 13g

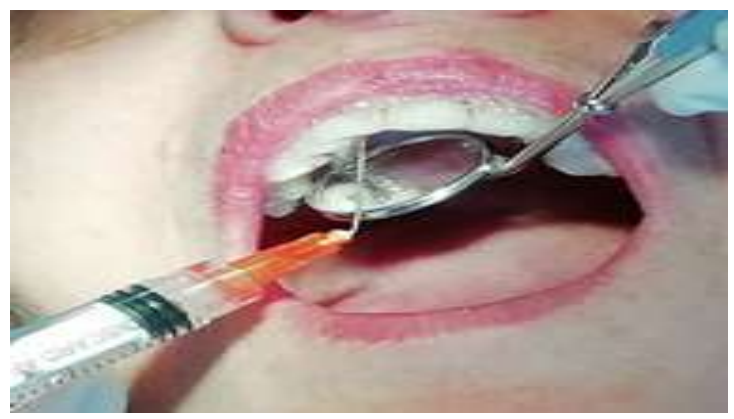

Figure 13h

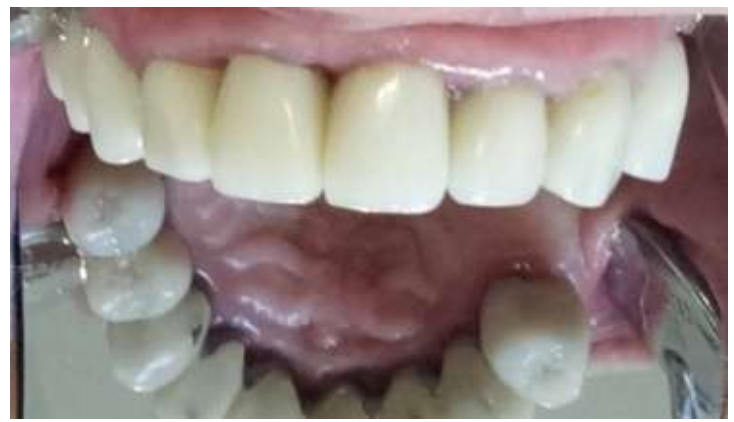

Figure 13i

Figure13g; 13h; 13i. Clinical check 24 months after the placement of the compression bridge with irrigation using an antiseptic combined with hydrogen peroxide

\section{DISCUSSION}

KERSCHBAUM and al (1991) reported an average lifespan of 15 to 25 years for conventional bridges and crowns. The average functional durability of removable prostheses would be 8 to 10 years. For this reason, one should always, during a prosthetic restoration, set the objective of making a fixed prosthesis, as far as possible [3]. Indeed, this is what motivated our therapeutic decision in front of the patient's will to keep her teeth; we opted for a fixed anterior and removable fixed prosthetic treatment to compensate for distal edentulousness in the maxilla and total in the mandibular arch.

The residual anterior teeth were too vestibulated and flattered; a bridge made on its abutments with a reduced periodontium therefore requires a significant reduction in the dental organ, which is why endodontic treatments and restorations with glass fiber posts were recommended in the preprosthetic phase. vital occur more often than on the living abutment teeth according to Lindhe and Nyman [4] We were motivated by the advantages of stride reconstructions with fiber posts, namely bonding to dental structures, good stress distribution similar to an unconstituted tooth, and moreover the protocol is conservative (no undercutting, no connection cone) which will significantly reduce the risk of fracture [5].

For psychological and especially aesthetic reasons, the cervical limit of the compression bridge was intra sulcular on the vestibular sides which would allow a gain of retention, a strapping effect for the reconstituted teeth whereas the literature however recommends cervical limits above. Gingival for the abutment teeth with reduced periodontium to facilitate the patient's control of bacterial plaque. [6]

According to Nyman, loss of retention is the most common cause of failure, especially for bridges with long pontics and ends without terminal abutments or extensions and which comes in the form of loosening followed by bridge fracture [7].

The occlusal regimen adopted was bilaterally balanced occlusion due to the presence of total mandibular assistant prosthesis. The compression bridge thus designed is very slightly stressed during the mandibular kinematics of protrusion and laterotrusion. According to Lundgren, patients with periodontal disease require not only periodontal therapy but also appropriate prosthetic 
rehabilitation. Contrary to traditional claims, clinical investigations published over the past 2 decades show that if the periodontium has been treated and rehabilitated, the fixed prosthesis can be performed on a reduced amount of periodontium, with a good long-term prognosis. It has also been shown that a significantly reduced but healthy amount of periodontium supporting such constructions can withstand considerable occlusal forces. [8]

Regarding the mobility of the pillars, Nayman found no mobility over 8 years of monitoring. Indeed, the total area of the periodontal ligament around the abutment teeth of 60 bridges inserted in patients who have been treated with periodontal disease was calculated and compared to the total area of the periodontal ligament of the teeth replaced by the bridges. Only $8 \%$ of these prosthetic restorations the area of the periodontal ligament of the abutment teeth was equal to or greater than that of the replaced teeth. In 57\% of cases, the area of the periodontal ligament of the abutment teeth was less than $50 \%$ of its normal initial value. Although periodontal support for restorations was greatly reduced, all bridges provided adequate functional restoration for 8-11 years. [9]

We ensured periodic checks of 3 months in the medium term for three years associated with medicinal irrigations, the periodontal tissues around the abutment teeth had not caused new losses of attachment during this maintenance period.

In addition Freilich et al tested the effect of fixed prostheses on hyper mobile abutment teeth with a substantially reduced periodontal support. An abutment tooth and a control tooth (nonabutment) of the same type and same periodontal condition were selected for a study on adult subjects.The treatment consisted of periodontal therapy, placement of a fixed prosthesis in three or four elements, and active participation during a 3-month maintenance. The 2-year data on 9 subjects revealed no significant difference (less than 1\%) between the abutment tooth and the control tooth. [10]

In 2007, a meta-analysis concluded that the bridge had a survival rate of $92.9 \%$ at 10 years, despite pillars that did not comply with Ante's law. [8.9]
To date, a good number of scientific works confirm that the success of a rehabilitation by the joint prosthesis of strongly reduced teeth and presenting a weakened periodontium is possible on condition of carrying out a scrupulous selection of the candidates (oral hygiene of the patient impeccable), to implement an adequate periodontal pre-prosthetic treatment, and to be able to ensure regular therapeutic follow-up in an individually adapted maintenance program. [8]

\section{CONClusion}

The durability of a joint prosthesis on a reduced periodontium depends on many factors associated with the tooth and the patient.

To be optimal, treatment must be comprehensive while respecting the various therapeutic approaches (pre-prosthetic, prosthetic and post-prosthetic treatments)

Under these conditions, the long-term effectiveness of the treatment can be obtained.

\section{BIBLIOGRAPHY}

[1] Dhenain Julien. Contention on reduced periodontium. Thesis university of Toulouse N³036; March 2017.

[2] Drain Maxime. Influence of occlusal desorders on the evolution of diseases periodontal Thesis university of Lille $\mathrm{N}^{\circ} 0370$ june 2018

[3] Daniel König Römerstrasse ZTM, Stephan Kickhöfen Mund und Werk Sasbacherstrasse ; Restauration prothétique fixe d'une denture au parodonte affaibli, Rev Mens Suisse Odontostomatol, Vol 110, Feb 2000.

[4] Lindhe J, Nyman S. A longitudinal study of combined periodontal and prosthetic treatment of patients with advanced periodontal disease. $\mathrm{J}$ Peridontol . Avr 1979;50(4):163-9.

[5] PLARD Hervé. Reconstitution coronoradiculaire. Université Rennes 1. Fiche de cours $\mathrm{n}^{\circ} \quad 3, \quad 2019$.disponible sur http://aecdr.net/uploads_o3/s1_ue2_ec3_frappe 4.pdf.

[6] Lundgren D: Prosthetic reconstruction of dentitions seriously compromised by periodontal disease. J Clin Periodontol 1991; 18: 390-395.

[7] Nyman S, Ericsson I. The capacity of reduced periodontal tissues to support fixed bridge work. J Clin Periodontol. sept 1982;9(5):40914.

[8] Freilich MA, Breeding LC, Keagle JG, Garnick JJ., Fixed partial dentures supported by 
periodontally compromised teeth, J Prosthet Dent. 1991 May;65(5):607-11.

[9] Lulic M, Brägger U, Lang NP, Zwahlen M, Salvi GE. Ante's (1926) law revisited: a systematic review on survival rates and complications of fixed dental prostheses (FDPs) on severely reduced periodontal tissue support.
Clin Oral Implants Res. juin 2007;18 Suppl 3:63-72.

[10] Ante's law. In: Wikipedia, the free encyclopedia [Internet]. 2013 [cité 5 oct 2016]. Disponible sur: https://en.wikipedia.org/w/index.php? title $=$ Ante $\% 27$ s_law\&oldid $=587577015$

Citation: Ait Mehdi. M, Zenati. L, Mebarki. S, Kehila. Bourouis.Y. Fixed Dental Prostheses on Reduced Periodontal Tissue Support: About a Case. ARC Journal of Dental Science. 2020; 5(2):11-18. DOI:https://doi.org/10.20431/2456-0030.0502003.

Copyright: (C) 2020 Authors. This is an open-access article distributed under the terms of the Creative Commons Attribution License, which permits unrestricted use, distribution, and reproduction in any medium, provided the original author and source are credited. 